\title{
Dynamic versus static:
}

\section{Evolving mechanical characterisation}

Materials used for aerospace applications have to be incredibly robust. For

commercial airlines, the materials to be capable of withstanding temperatures from -45 to $45^{\circ} \mathrm{C}$ kllomertres per hour. All materials designed to replicate the real dynamic conditions encountered during use. Dr Meysam Rahmat, in his research at the Aerospace Research Council Canada, works accurately measure and test the types of dynamic forces such use to better understand their behaviour and properties. used to build the aircraft need and windspeeds of over 400 Research Centre of the National on designing new tests to more

\begin{abstract}
erospace materials are some
of the most stringently tested
materials on earth. They have ch withstanding a wide range of environmental conditions, such as temperature and pressure, while retaining their desired properties. While durability of such materials is mandatory, it is even more important that failures of the material are easily, and sufficiently is to prevent accidents such as the Chin Airlines Flight 611 crash, where metal fatigue led to the deaths of 225 people.

Being able to detect impending failure of materials requires a comprehensive knowledge of the material's behaviour. To do so, these materials are studied using a variety of lab-based testing techniques, alongside numerical simulations, in an effort to predict how the material behaves close to the failure point.
\end{abstract}

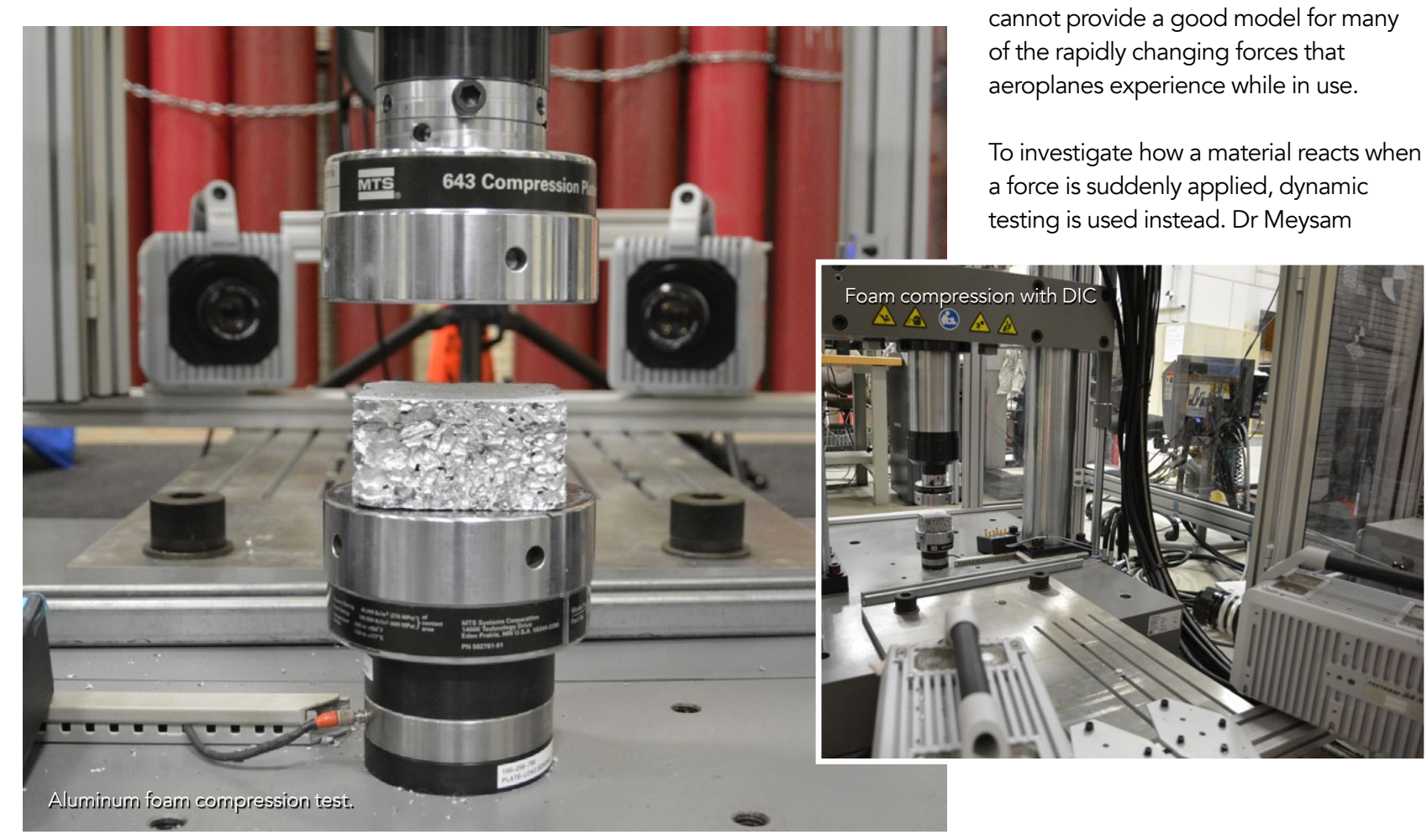

For such testing and modelling to be useful for aerospace materials, they must effectively mimic the types of forces the material would experience when in use. For example, the metals used for aeroplane wings are tested in the lab once they are made into wings by bending them and applying stress and strain to the materials on a variety of testing rigs. Through repeated understanding of the a comprehensive be achieved to identify what ealy warning signs can be used for in-situ testing once the aeroplane is in use.

Many of the existing tests look at applying static forces to the material. This is where the load applied to the material is essentially constant over a given time and would be a good way of modelling, for example, the constant force that the structure. However, static stress tests a good mode for eroplanes experience while in use.

To investigate how a material reacts whe a force is suddenly applied, dynamic ing is used instead. Dr Meysam

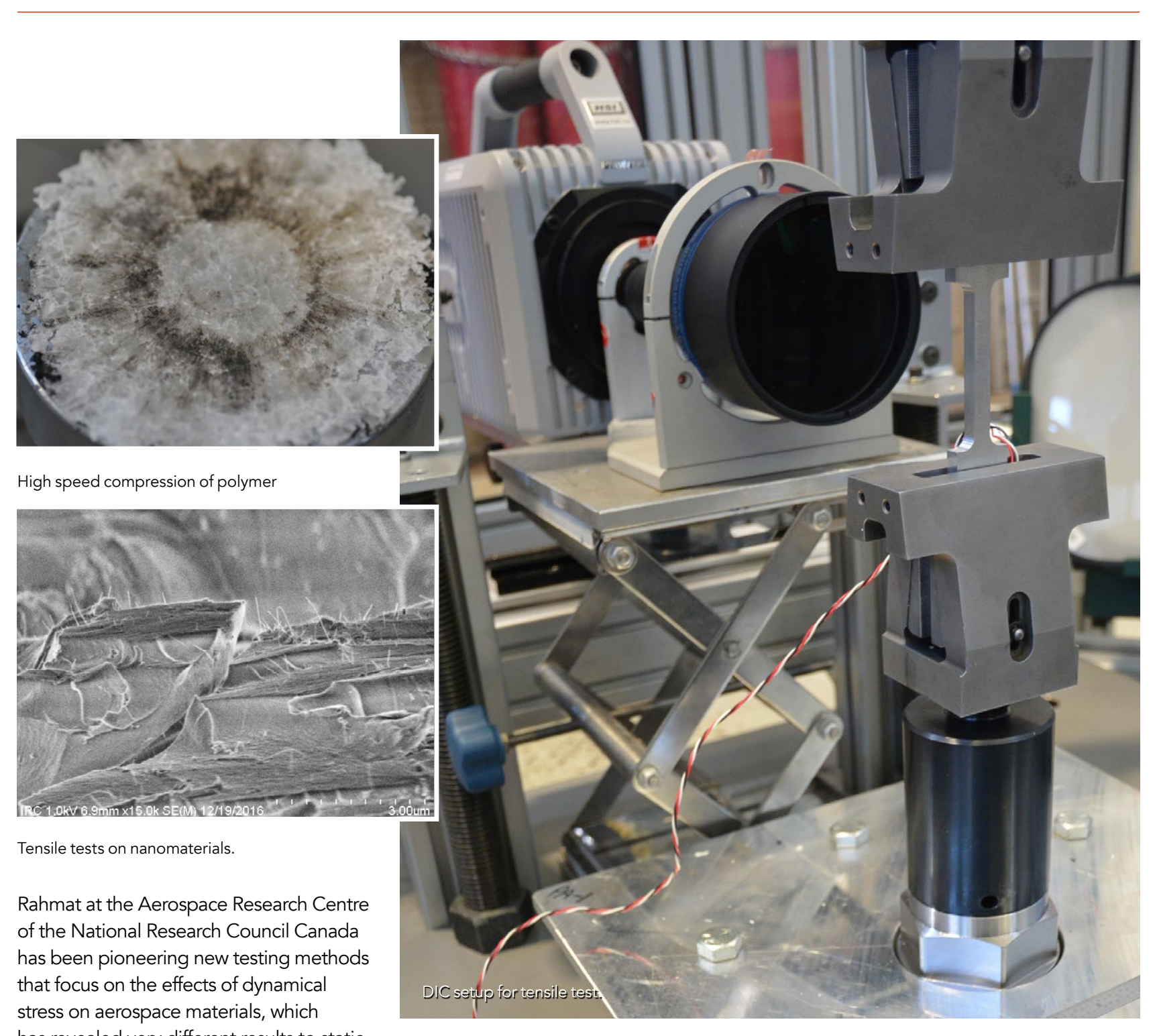

stress on aerospace

has revealed very different results to static or quasi-static (where the force is applied over a longer period of time than it wo implications for aircraft safety.

\section{STRESSFUL MEASUREMENTS} To perform the dynamic strain tests, Drame. Such frames can be customised for different types and magnitudes of load. For Dr Rahmat's dynamic tests, he uses a load frame specifically designed to achieve high speeds. The frame can reach speeds of up to eight metres per second as it suddenly applies load to the stationary sample, resulting in the sudden transfer of force. A series of sensors measure local deformation in different locations on the sample to obtain 'stress' (a measure of force acting on the sample that can lead to deformation) and 'strain' (a measure of deformation in the materia
Exclusively using static tests cannot provide a good model for many of the rapidly changing forces that aeroplanes experience while in use.

One common issue with these types of dynamic stress measurements is that the values measured by the load cell oscillate during the measurement, making it difficult to extract the true stress-strain values experienced by the sample. Some preventive actions, such as using lightweight clamps and optimising the can carried out to minimise these oscillations. Part of Dr Rahmat's work has been to carefully investigate how changing testing parameters such as sample geometry the material properties to ensure their deformation. This is important for ease of accuracy and whether these corrections to minimise the oscillations are applicable to different materials.

\section{STESS FRACTURES}

rials Dr Rahmat has investigated using his approach is an aluminium dogbone structure, named for its resemblance to the shape of a dog bone. This is a typical shape for a tensile test sample as the narrow section in the middle is subject to the majority of the testing: if the stresses were concentrated 


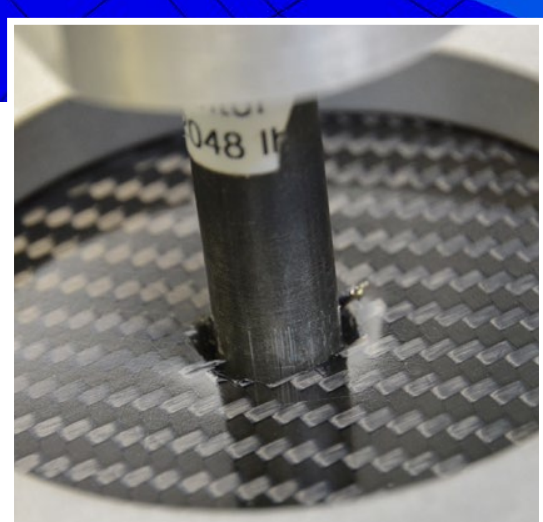

Puncture test on composites

is clamped to the testing machine, this would lead to artificial readings as the clamping of the sample would interfere with the measurement. This is also beneficial for measuring elongation, as means the measured change in length of

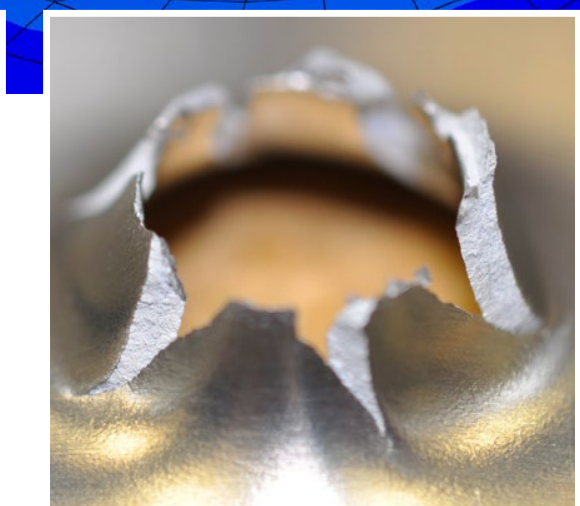

Punctured aluminum.

type of fractures the material experienced depending on whether it had received the force very suddenly or gradually over a longer period. While small changes in the surface structure of the metal that can only be observed by a microscope might

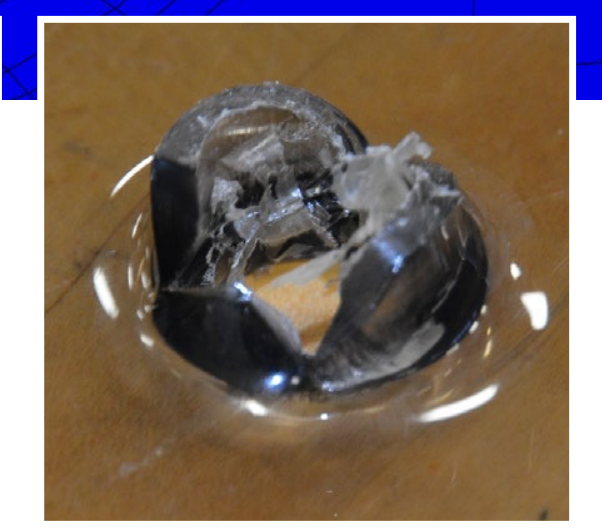

Puncture test on polymer.

nanoscale materials and have not been well-studied using dynamic strain methods. By testing a nanocomposite formed of a common epoxy, with and without boron nitride nanotubes, $\mathrm{Dr}$ Rahmat and his team were able to see how the inclusio exclusively from the material, not in the clamps.

The information obtained from such tests is highly valuable not just of the nanotubes or understanding material behaviour elasticity and tensile elasticity and ten
strength to the little influence on During these but also from a design perspective.

little influence on

the shear properties of the epoxy.

Dr Rahmat found was that for this wellstudied aluminium sample, there was a large deviation for the ultimate streng and the elongation at failure between the values measured dynamically ersus quasi-static measurement. This is important for not just making sure the testing of materials is realistic for what it will ultimately experience in the field, but also refining the modelling of the responses of such materials

Optical microscope images of the tested seem trivial, this indicates differences in the amount of energy absorption by The information obtained from such the aluminium depending on the speed of the force, showing the material would have different resistances to different types of loading conditions.

METALS TO NANOCOMPOSITES All of this testing and model refinement has ultimately enhanced our understanding of very well-studied materials, but Dr Rahmat has been expanding this to nanocomposites complex materials composed of different understanding material behaviour but also from a design perspective, as it becomes have the most desirable properties. The methods Dr Rahmat has been developing are also very generalisable to a wide range of substances, which he
will continue to explore in his future work.

\section{(-) Behind the Research}

Dr Meysam Rahmat

E: meysam.rahmat@nrc-cnrc.gc.ca $\quad$ T: +1 (613)991 $5360 \quad$ W: www.nrc-cnrc.gc.ca/eng/

\section{Research Objectives}

Dr Meysam Rahmat's work focuses on dynamic mechanical Dracterisation of materials, particularly nanoco

\section{Detail}

Aerospace Research Centre

Canada

1200 Montreal Road
Ottawa, ON

Ottawa, ON

Canada

Bio

Dr Rahmat received his $\mathrm{PhD}$ in mechanical engineering from McGill University in 2011, and started working at the National Research Council Canada (NRC) the following year His main focus is on composite materials, nanocomposites,

unding

earch Council Canada through Security Materials Technology Program, Project A1-006934

\section{Collaborators}

- Richard Desnoyers

- Behnam Ashrafi

- David Backman

- Gang Li

- Michael B. Jakubinek

- Yadienka Martinez-Rubi

Benoit Simard: National Research Council Canada

\section{Canadà ncesico}

References

Meysam Rahmat, "Dynamic Mechanical Characterization of Aluminum: Analysis of Strain-Rate Dependent Behavio ", Mechanics of Time-Dependent Materials, 2018, In Press, https://doi.org/10.1007/s11043-018-9393-0.

Meysam Rahmat, Alex Naftel, Behnam Ashrafi, Michael B. Jakubinek, Yadienka Martinez-Rubi, Benoit Simard, "Dynam "Epoxy Nanocomposites", Polymer Composites, 2018, In Press, https://doi.org/10.1002/pc.24995.

\section{Personal Response}

\section{What types of material do you want to explore next}

\section{with this testing method?}

II Any type of homogeneous (including metals, polymers and ceramics) and non-homogeneous material (including composite materials) can be characterised using this as closed cell foams are currently being tested to as closed cell foams are currently being tested to evaluate
their energy absorption capabilities under impact. What is the most satisfying aspect of your work in this field?

II Mechanical characterisation is an essential step in the iterative process of structural design; whether through proo of concept or screening tests in the earlier stages of the process, or daring fill scale winal evaluation of the structure is to ensure that during its service life, the structure will not experience any catastrophic fallure after going through all those harsh experiments in the lab. When an aircraft retires after many years of service with no major problem, multi-disciplinary system worked to its best and served its function. That's when an engineer's effort is paid off. Also, there is no denying that crashing and breaking lab
specimens at high velocity is fun! 